\title{
O nicho trófico de duas espécies simpátricas de Aegla Leach (Crustacea, Aeglidae) no tributário da bacia hidrográfica do Rio Pelotas, Rio Grande do Sul, Brasil ${ }^{1}$
}

\author{
Thais Castro-Souza ${ }^{2} \&$ Georgina Bond-Buckup ${ }^{2}$ \\ ${ }^{1}$ Contribuição $n^{\circ} 443$ do Departamento de Zoologia, Universidade Federal do Rio Grande do Sul. \\ 2 Programa de Pós-Graduação em Biologia Animal, Departamento de Zoologia, Instituto de Biociências, Universidade Federal \\ do Rio Grande do Sul. Avenida Bento Gonçalves 9500, prédio 43435, 91501-970 Porto Alegre, Rio Grande do Sul, Brasil.
}

\begin{abstract}
The trophic niche of two sympatric Aegla Leach species (Crustacea, Aeglidae) in a tributary of hydrographic basin of Pelotas River, Rio Grande do Sul Brazil. Freshwater anomurans of the Aeglidae are restricted to tropical and temperate South America. Some of the more than 60 known species are sympatric, among them Aegla camargoi (Buckup \& Rossi, 1977) and Aegla leptodactyla (Buckup \& Rossi, 1977), occur in the mountainous region of Rio Grande do Sul, the southernmost Brazilian state. The interactions between these two sympatric species, especially sharing of food resources, is still largely unknown; this is the reason this study was done. Samples of each species were collected at 6 and 12 hour intervals during spring, summer, autumn and winter. The degree of fullness of their stomachs was calculated by the point method, frequency of occurrence of different types of food debris and feeding index. The breadth of the niche was estimated for each species using Levins' Index and the niche overlap with Pianka's and Schoener's methods. Both species fed principally on macrophytes and immature insects of Ephemeroptera, Trichoptera, Coleoptera and Diptera. They feed anytime of the day with no seasonal differences. Although the breadth of the trophic niche of each species showed small seasonal variations, the trophic niches of the two species overlapped during all seasons. These results suggest that these species are generalist omnivores and share food resources available in the environment.

KEY WORDS. Coexistent species, Decapoda, diet, niche overlap.
\end{abstract}

RESUMO. Os anomuros de Aeglidae são restritos às águas doces da região subtropical e temperada da América do Sul. Entre as mais de 60 espécies conhecidas da ciência, algumas são simpátricas, destacando-se Aegla camargoi Buckup \& Rossi, 1977 e Aegla leptodactyla Buckup \& Rossi, 1977, que ocorrem na região serrana do Rio Grande do Sul, Brasil. A interação das duas espécies simpátricas, especificamente o compartilhamento dos recursos alimentares, ainda permanece desconhecido. Nesta investigação, a alimentação natural, a largura e a sobreposição de nicho trófico das espécies são estudadas e comparadas. Os animais foram coletados sazonalmente, em intervalos de 6 por 24 horas. Em laboratório, foi analisado o conteúdo estomacal, utilizando as técnicas do método dos pontos, da classificação do grau de repleção estomacal, da frequiência de ocorrência e o índice alimentar. A largura de nicho de cada espécie foi estimada, segundo o Índice de Levins. Para estimar a sobreposição de nicho, utilizaram-se duas medidas de sobreposição, de Pianka e de Schoener. As duas espécies alimentam-se preferencialmente de macrófitas, e insetos imaturos das ordens Ephemeroptera, Trichoptera, Coleoptera e Diptera, entre outros itens. A estimativa de repleção estomacal indicou que as duas espécies alimentam-se em todos os horários, sem diferenças entre as estações do ano. A largura do nicho trófico de cada espécie revelou pequenas variações sazonais. A sobreposição de nicho trófico entre as duas espécies ocorre em todas as estações do ano. Os resultados sugerem que as espécies são omnívoras generalistas, partilhando os recursos disponíveis no ambiente. PALAVRAS CHAVE. Decapoda, dieta, espécies coexistentes, sobreposição de nicho.

A ocorrência de espécies simpátricas entre os aeglídeos é um fenômeno freqüente em cursos d'água dos estados sul-brasileiros, tendo registros de nove pares de espécies (BOND-Buckup 2003).
Entre estas, destaca-se a coexistência de Aegla camargoi Buckup \& Rossi, 1977 e Aegla leptodactyla Buckup \& Rossi 1977 em tributário do Rio Pelotas, formador da Bacia do Rio Uruguai. 
Nos estudos sobrea dieta natural de crustáceos decápodes, tanto o método utilizado no exame do conteúdo estomacal como a caracterização temporal, nem sempre é possível conhecêla em observações somente no campo (WILLIAMs 1981). Considerando os hábitos da maioria das espécies dos caranguejos anomuros do gênero Aegla Leach, 1920 que vivem embaixo de pedras no substrato dos cursos d'água no sul da América do Sul (Bond-Buckup \& Buckup 1994), torna-se inviável a caracterização trófica em seu hábitat. Este fato pode ser constatado em certos microhábitats de algumas espécies de aeglídeos, como no Rio da Divisa, no nordeste do Rio Grande do Sul, com lajes densamente cobertas por macrófitas Podostemonaceae, onde A. leptodactyla se al imenta e os juven is se abrigam (Noro \& Buckup 2002).

As pesquisas que abordam a dieta de aeglídeos são ainda incipientes, considerando o número de espécies conhecidas. Destacam-se as informações sobre a espécie Aegla laevis (Latreille, 1818), em território chileno, que alimenta-se, entre outros itens, de plantas submersas e de Oligochaeta (BAHAM ONDE \& LoPEZ 1961). Na gruta da Peroba, em São Paulo, A egla perobae Hebling \& Rodrigues 1977 foi considerada primordialmente carnívora, alimentando-se sobretudo de insetos vivos que caem na água elarvas de insetos aquáticos (RodRIGUES \& HeBLING 1978). Recentemente, BUENo \& BOND-BuCKuP (2004) informam sobre a dieta natural de Aegla platensis Schmitt e Aegla ligulata BondBuckup \& Buckup, espécies que se encontram em diferentes altitudes. As autoras verificaram uma gran de diversidade de itens al imentares no estômago dos exemplares das duas espécies, incluindo, entre outros, al gas e plantas vasculares, invertebrados como Insecta, escamas de peixes e, ainda, grãos de areia.

Em contraste com os aeglídeos, os resultados sobre a dieta natural de crustáceos decápodos vem sen do bem documentada, em Brachyura (WILliams 1981, 1982, WEAR \& HADDON 1987, Haefner 1990, Mantelatto \& Petracco 1997, Mantelatto \& ChristofolettI 2001, Branco \& Verani 1997, Brogim \& Lana 1997, Kapusta \& Bemvenuti 1998).

Por outro lado, o conhecimento das relações tróficas entre espécies possibilita uma melhor compreensão dos mecanismos que permitem que espécies proximamente relacionadas explorem o mesmo nicho (RosAs et al. 1994) e constituem robustas ferramentas queseapoiam em conceitos da teoria ecológica (CHARVET et al. 1998). Estudos sobrea largura e sobreposição denicho trófico são importantes para quantificar como duas espécies se sobrepõem quanto à utilização dos recursosal imentares (HURLBERT 1978, Albertonı et al. 2003), ou mesmo para ser usada como uma medida descritiva da organização da comunidade (ABRAms 1980). A sobreposição, por outro lado, pode evidenciar ausência de competição, se um dos recursos é superabundante ou então é irrelevante para uma das espécies (Colwell \& Futuyma 1971).

Neste estudo propõe-se caracterizar a ecologia trófica de duas espécies simpátricas, Aegla camargoi e Aegla leptodactyla, com ênfase em seus hábitos alimentares, verificando a largura e a sobreposição de nicho trófico.

\section{MATERIAL E MÉTODOS}

\section{Á rea de estudo e coleta dados}

Foram realizadas coletas sazonais, nos meses de outubro/ 2001, janeiro, abril e julho/2002, no curso superior do Rio da Divisa, (28³8'17"S, 4957'56"W), tributário da margem esquerda do Rio Pelotas, município de São José dos Ausentes, Rio Grande do Sul. As coletas ocorreram em quatro horários no ciclo de 24 horas: às 6, 12, 18 e 24 h. Em cada horário foram coletados, manualmente com auxílio de puçás, 10 exemplares adultos de cada espécie, sendo cinco machos e cinco fêmeas. A temperatura da água foi registrada às $12 \mathrm{~h}$. A medida de comprimento do cefalotórax de cada animal foi obtida, através de paquímetro digital. O material foi fixado imediatamente em formalina tamponada a $10 \%$, e analisado em laboratório. Cumpre salientar que os indivíduos das duas espécies foram coletados juntos, sugerindo que ambas ocorrem no mesmo micro-habitat.

\section{A nálise do conteúdo estomacal}

A carapaça dos animais foi seccionada junto à margem dorsal, rebatida e o estômago retirado. Foram utilizados apenas exemplares em intermudas, sendo rejeitados os indivíduos em ecdise, com carapaça mole, como sugerido por HaEFNER (1990).

O grau de repleção estomacal (GR) foi determinado visualmente, adotando os procedimentos de WILLIAMS (1981), seguindo uma escala de seis classes:

Classe 1 ( $0 \%$ - vazio); classe 2 ( $<5 \%$ - parcialmente vazio); classe 3 (5 a 35\% - vazio/ médio); classe 4 (>35 a 65\% médio); classe 5 (>65 a 95\% - médio/ cheio), e classe 6 (>95\% - cheio).

Utilizou-se os métodos de freqüência de ocorrência (FO) e dos pontos (MP), (WILliams 1981, Wear \& HadDon 1987, Haefner 1990, Branco \& Verani 1997), complementados pelo índice alimentar (IA) de KaWAKamI \& VAZzoler (1980).

A freqüência de ocorrência (FO) foi definida como o número de indivíduos cujos estômagos continham determinado item i, dividido pelo número total de indivíduos amostrados.

No método dos Pontos (MP), a contribuição relativa de cada item, no volume total de alimento em um determinado estômago, foi determinada em uma escala de pontos com cinco graus. A porcentagem do volume que cada item ocupou no estômago foi estimada utilizando-se uma placa de Petri com papel milimetrado em seu fundo (Hollows et al. 2002). Se determinado item ocupou $<5 \%$, recebeu 2,5 pontos; de 5 a $35 \%$ $=25$ pontos; $>35$ a $65 \%=50$ pontos; $>65$ a $95 \%=75$ pontos, e $>95 \%=100$ pontos.

O número total de pontos para cada item alimentar foi atribuído conforme o grau de repleção. Calculou-se multiplicando o número de pontos de cada item pelo valor do grau de repleção do estômago, de acordo com a classe: GR classe $1=$ 0,00 ; GR classe $2=0,02$, GR classe $3=0,25$; GR classe $4=0,50$; GR classe $5=0,75 ; \mathrm{GR}$ classe $6=1,00$ 


\section{Índice A limentar (IA)}

Nessa análise, foram combinados os dois métodos, o da freqüência de ocorrência e o método dos pontos, adotando-se as recomendações de KaWAKAmı \& VAZzoler (1980), para efeito de comparação e mel hor compreensão da importância de cada categoria trófica: IA =FO.MP/ $\Sigma$ (FO.MP), onde IA =índice alimentar; $F O=$ freqüência de ocorrência do item $i$; $M P=$ pontos do item i (\%).

\section{Largura e sobreposição de nicho}

A largura de nicho das duas espécies foi estimada pelo Índice de Levins, resultado de uma adaptação do Índice de Diversidade de Simpson (Recíproco de Simpson) (Levins 1968): $\mathrm{B}=1 / \Sigma \mathrm{Pj}^{2}$, onde $\mathrm{B}=$ medida de largura de nicho de Levins; $\mathrm{Pj}=$ proporção de indivíduos utilizando o recurso j. O valor de $B$ pode variar de 1 a $n$, onde $n$ é o número de recursos al imentares presentes nos estômagos.

A sobreposição de nicho foi estimada baseando-se nos métodos de: 1) PIANKA (1973) o qual fornece valores de sobreposição de nicho entre 0 e 1; a sobreposição entre a espéciej ek é igual a sobreposição entre a espécie $\mathrm{k}$ e j: Ojk $=\Sigma \mathrm{Pij} \times \mathrm{Pik} /$ $\mathrm{V} \Sigma \mathrm{Pij}^{2} \Sigma \mathrm{Pik}^{2}$, onde Ojk = medida de sobreposição de Pianka entre as espécies j ek; Pij, Pik =proporção do recurso i em um total de recursos utilizados pela espécie j, e pela espécie k; e 2) Sobreposição de SchoENER (1970), expressa em porcentagem, é dada pela fórmula: $\mathrm{Pjk}=[\Sigma($ mínimo Pij, Pik) $] \times 100$, onde Pjk = porcentagem de sobreposição entre j e k; mínimo Pij, Pik =menor valor da proporção de recursos entre a espéciej ek. Estes índices são os mais indicados para análise da sobreposi ção de nicho trófico, uma vez que se apóiam nas proporções dos itens al imentares encontrados nos estômagos (KREBS 1989).

Em todos os testes estatísticos adotou-se $p \leq 0,05$. O teste do $\chi^{2}$ foi aplicado à freqüência percentual relativa em pontos dos taxa de presas, material digerido e areia, de machos e fêmeas de cada espécie. Os itens al imentares ingeridos por machose fêmeas de ambas espécies não apresentaram diferença significativa na proporção, eforam, portanto, agrupados. Também utilizou-se o teste $\chi^{2}$ para comparar os val ores das larguras de nicho de ambas espécies. A análise estatística ANOVA foi utilizada para detectar possíveis diferenças significativas entre os valores mé dios de GR de cada sexo, nos quatro horários amostrados, e nas quatro estações do ano. O teste t foi utilizado para detectar possíveis diferenças significativas entreo GR médio entre machose fêmeas, nos horários de coleta e estações do ano.

\section{RESULTADOS}

A área de estudo localizada no Planal to Sul Riograndense, representa um ambiente de especial interesse ecológico, pois nesta região encontram-se as nascentes do Rio Pelotas. O clima da região é caracterizado como mesotérmico médio, com invernos acentuados e verões brandos pelo efeito da altitude, com registro de temperatura média anual entre 12 e $14^{\circ} \mathrm{C}$, no inverno a média das mínimas temperaturas diárias mantém-se abaixo de $6^{\circ} \mathrm{C}$ (NIMER 1989).

No período amostrado, no entanto, a temperatura da água registrada no Rio da Divisa, mostrou o menor valor no inverno, $9^{\circ} \mathrm{C}$. Na primavera a temperatura registrada foi de $19^{\circ} \mathrm{C}$; no verão atingiu a maior temperatura, $22,1^{\circ} \mathrm{C}$ e, finalmente, no outono verificou-se uma temperatura da água de $12,8^{\circ} \mathrm{C}$. Os exemplares amostrados apresentaram uma variação de comprimento de cefalotórax de 7,16 mm a 31,62 mm em Aegla camargoi, e de $8,87 \mathrm{~mm}$ a 22,93 mm em Aegla leptodactyla. Entretanto, não foi observada variação na composição da dieta em relação ao tamanho dos animais, isto é, indivíduos pequenos freqüentemente apresentaram estômagos repletos de partes de imaturos de Insecta.

\section{Grau de repleção estomacal (GR)}

Foram analisados 147 estômagos de A. camargoi. Destes, apenas $1(0,7 \%)$ estava vazio e $146(99,3 \%)$ apresentaram conteúdo. Dos estômagos com conteúdo, 80 (54,8\%) eram de machos adultos e $66(45,2 \%)$ de fêmeas adultas. Em A. leptodactyla analisou-se 160 estômagos, destes, encontrando-se apenas cinco vazios (6,25\%). Dos estômagos com conteúdo, 77 (49,7\%) eram de machos e 78 (50,3\%) de fêmeas.

A variação sazonal do grau médio de repleção (GR médio) de A. camargoi e A. leptodactyla nos quatro horários de coleta, é apresentada na figura 1. Não foram detectadas diferenças significativas de grau de repleção médio de machos e fêmeas entre os horários de coleta e as estações $(p \leq 0,05)$. Da mesma forma, não foram detectadas diferenças significativas no valor de repleção estomacal média entre as duas espécies estudadas nas diferentes estações do ano $(p \leq 0,05)$.

Pela análise da variação diária do GR médio em $A$. camargoi e A. leptodactyla, pode verificar-se que os estômagos estiveram de médio a cheios em todos os horários, o que não permitiu uma clara detecção do horário preferencial dealimentação (Fig. 2). Os dados obtidos mostraram que não há diferença significativa nos horários e entre os valores diários de grau de repleção das duas espécies.

\section{A mplitude trófica}

Quanto à composição da dieta, 15 categorias alimentaresforam identificadas para A. camargoi, e 14 paraA. leptodactyla, incluindo material animal, vegetal, sedimento e material digerido. Os itens alimentares presentes nos estômagos foram os mesmos para as duas espécies, à exceção de Ostracoda e Oligochaeta, encontrados apenas em A. camargoi, eo Platyhel minthes Temnocephala sp., encontrado apenas em A. leptodactyla.

Pela da análise de freqüência de ocorrência (FO), os itens mais freqüentes nos estômagos deA. camargoi foram macrófitas, material digerido, incluindo o material orgânico não identificado, e partes de insetos não identificados. Os insetos identificados foram constituídos, na sua totalidade, de estágios imaturos, como larvas e pupas. Nos estômagos de A. leptodactyla, por sua vez, os itens mais freqüentes foram macrófitas, partes de insetos, e material digerido. 


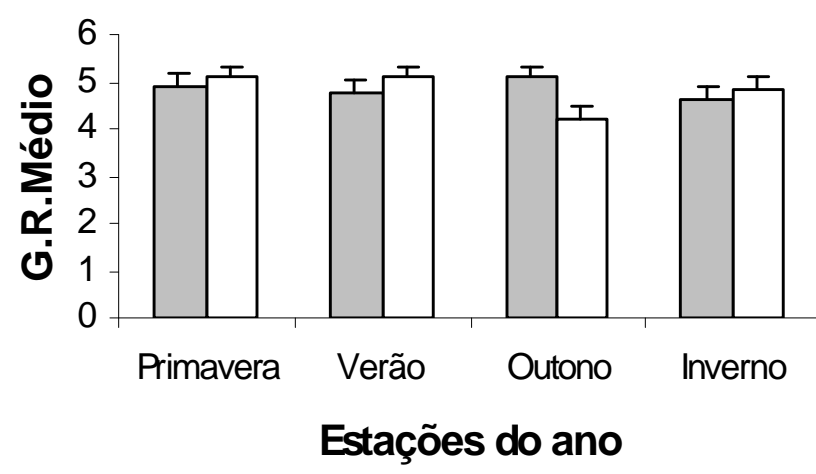

Aegla camargoi

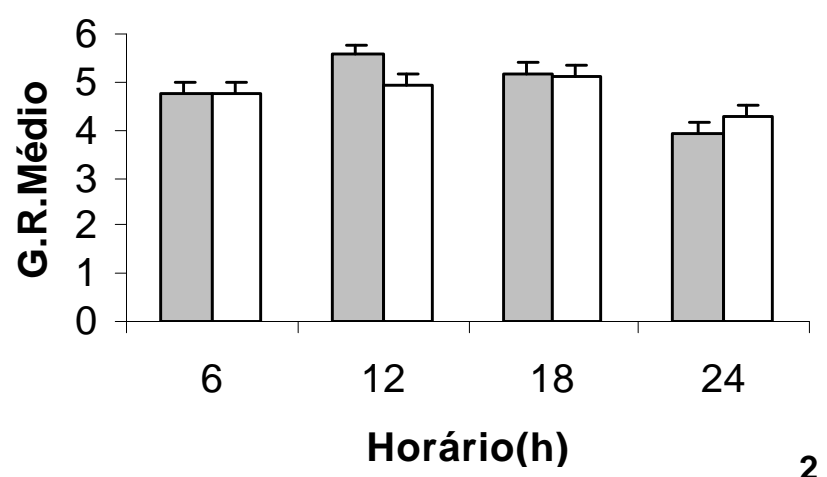

$\square$ Aegla leptodactyla

Figuras 1-2. Aegla camargoi e Aegla leptodactyla: (1) variação sazonal e (2) diária do grau de repleção médio (e os respectivos erros padrões) dos estômagos dos indivíduos amostrados no Rio da Divisa, São José dos Ausentes, Rio Grande do Sul, na primavera/2001, verão, outono e inverno/2002.

Ao longo das quatro estações do ano, a dieta esteve composta pelas mesmas categorias alimentares. $\mathrm{Na}$ análise sazonal da FO e do IA dos principais recursos, verificou-se o predomínio de macrófitas em todas as estações, seguidos por Insecta, tanto em A. camargoi como em A. leptodactyla. A análise do IA confirmou como os itens mais importantes para as duas espécies, as macrófitas e Insecta, não levando em consideração o material digerido, que não pôde ter a sua procedência identificada. As macrófitas, identificadas como plantas vasculares, excluindo as algas, foram o principal componente nos estômagos, confirmando essa categoria como a mais importante para as espécies. Os insetos apresentaram baixos vaIores de IA, indicando que, apesar de freqüentes em todas as amostragens, ocuparam um pequeno volume no estômago, quando comparados às macrófitas, e seus pontos consequentemente foram mais baixos (Figs 3 e 4).

$\mathrm{Na}$ análise pelo método dos pontos (MP), os itens mais volumosos para A. camargoi foram equivalentes aos mais freqüentes. Em A. leptodactyla, os itens mais volumosos foram macrófitas, material digerido, partes de insetos, partes de crustáceos aeglídeos, e imaturos de Insecta Ephemeroptera (Tab. I). Os pontos das macrófitas representaram quase o triplo de pontos do material digerido, e cerca de seis vezes mais pontos que o terceiro colocado, as partes de insetos, indicando que elas são o principal recurso alimentar de A. leptodactyla, e os insetos atuariam como recurso secundário.

\section{Largura e sobreposição de nicho trófico}

Na comparação entre os valores de largura de nicho de A. camargoi e A. leptodactyla, observou-se valores semel hantes em todas as estações (Fig. 5). O teste estatístico $\chi^{2}$ aplicado às larguras de nicho de ambas espécies não detectou diferença significativa nas quatro estações do ano. Com relação à sobreposição de nicho, as duas estimativas indicaram altos valores entre as espécies. A medida de sobreposição de PIANKA
(1973) forneceu altos valores de sobreposição das espécies em todas as estações (Fig. 6). Neste estudo, o valor mais baixo pode ser observado no verão $(0,76)$, quando não se encontrou Insecta Coleoptera e Diptera nos estômagos de A. camargoi, e escamas de peixe e Insecta Ephemeroptera nos estômagos de A. leptodactyla. O mais alto valor de sobreposição foi observado no inverno $(0,98)$. O Índice de sobreposição de SCHOENER (1970) também mostrou valores altos em todas as estações. Segundo esse método, a maior sobreposição entre a utilização de recursos foi verificada no inverno, quando atingiu $83 \%$, e a menor no verão, 61\% (Fig. 6).

\section{DISCUSSÃ O}

A análise sazonal do GR mostrou para A. camargoi um pico de repleção no outono, e para $A$. leptodactyla, os maiores val ores foram na primavera e no verão. Cabe salientar que em todas as estações do ano os estômagos estiveram com repleção acima do grau médio, e os valores de GR foram muito próximos, não permitindo identificar uma preferência sazonal de alimentação. Em A. platensis, BuENo \& Bond-BUCKUP (2004) verificaram um maior valor de repleção estomacal no outono, que coincidiu com o período de maior diversidade de recursos alimentares disponíveis no ambiente. A. ligulata, por sua vez, apresentou um pico sazonal de grau de repleção na primavera. As duas espécies apresentaram estômagos relativamente cheios durante todo o ano.

Pela variação diária do grau de repleção obtida, $A$. camargoi apresenta atividade alimentar intensa em todos os horários, com um pico às $12 \mathrm{~h}$. Em A. leptodactyla, da mesma forma, ocorre uma atividade alimentar intensa em todos os horários, com maior atividade de alimentação às $18 \mathrm{~h}$. A. platensis apresentou maior $\mathrm{GR}$ às $24 \mathrm{~h}$, indicando uma tendência ao forrageio noturno, e A. ligulata, semel hante ao observado para A. leptodactyla, apresentou maior GR às 18 h (BUENo \& 


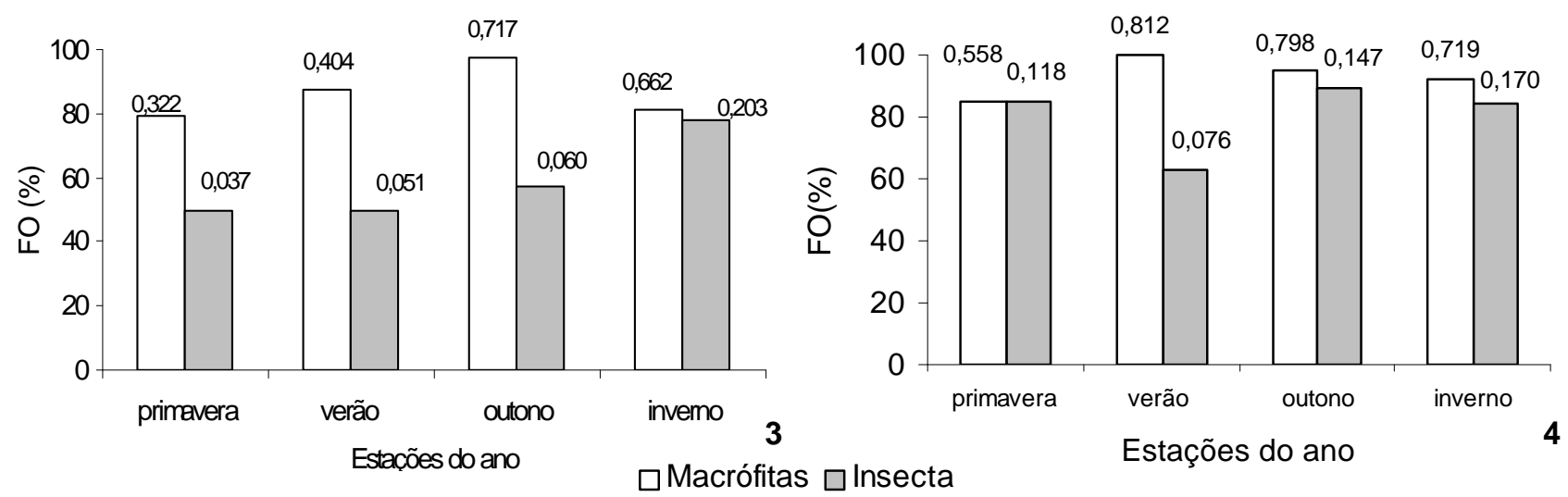

Figuras 3-4. Freqüência de ocorrência FO (\%) e os respectivos Índices alimentares (IA, números acima da barra) dos dois grupos mais importantes encontrados nos estômagos dos indivíduos amostrados no Rio da Divisa, São José dos Ausentes, Rio Grande do Sul, na primavera/2001, verão, outono e inverno/2002. (3) Aegla camargoi; (4) Aegla leptodactyla.

Tabela I. Aegla camargoi e A. leptodactyla. Método dos pontos (MP), freqüência de ocorrência (FO) e os respectivos índices alimentares (IA) dos itens alimentares encontrados nos estômagos dos indivíduos amostrados na primavera/2001, verão, outono e inverno/2002.

\begin{tabular}{|c|c|c|c|c|c|c|}
\hline \multirow{2}{*}{ Itens } & \multicolumn{3}{|c|}{ A. camargoi $(\mathrm{N}=147$, vazio $=1)$} & \multicolumn{3}{|c|}{ A. leptodactyla ( $\mathrm{N}=160$, vazio 5) } \\
\hline & M.P & F.O & IA & M.P. & F.O & IA \\
\hline Algae & 53,00 & 2,28 & 0,000160 & 32,87 & 3,18 & 0,0001100 \\
\hline Macrofitas & 4847,24 & 87,58 & 0,570000 & 7313,75 & 93,00 & 0,7700000 \\
\hline \multicolumn{7}{|l|}{ Platyhelminthes } \\
\hline Metacercária & 26,87 & 4,78 & 0,000170 & 10,00 & 3,25 & 0,0000370 \\
\hline Temnocephalidae & 0 & 0 & 0 & 2,50 & 0,62 & 0,0000020 \\
\hline Oligochaeta & 37,50 & 1,25 & 0,000063 & 0 & 0 & 0 \\
\hline \multicolumn{7}{|l|}{ Insecta } \\
\hline Partes de Insecta & 646,62 & 42,58 & 0,037000 & 1197,62 & 55,07 & 0,075000 \\
\hline Ephemeroptera & 222,75 & 7,50 & 0,002200 & 233,75 & 7,68 & 0,002000 \\
\hline Trichoptera & 58,75 & 5,00 & 0,000390 & 40,62 & 7,66 & 0,000350 \\
\hline Coleoptera & 21,25 & 1,25 & 0,000035 & 76,25 & 5,71 & 0,000490 \\
\hline Diptera & 78,00 & 2,28 & 0,000230 & 56,62 & 3,28 & 0,000210 \\
\hline Acarina & 2,50 & 1,03 & 0,000003 & 4,37 & 1,25 & 0,000006 \\
\hline \multicolumn{7}{|l|}{ Crustacea } \\
\hline Ostracoda & 18,75 & 0,62 & 0,000015 & 0 & 0 & 0 \\
\hline Aeglidae & 120,00 & 5,00 & 0,000810 & 311,25 & 11,68 & 0,004100 \\
\hline Escamas de peixe & 49,37 & 5,00 & 0,000330 & 15,00 & 1,25 & 0,000021 \\
\hline Areia & 409,24 & 27,08 & 0,015000 & 236,00 & 16,81 & 0,004500 \\
\hline Material digerido & 4394,49 & 62,86 & 0,370000 & 2281,76 & 53,07 & 0,140000 \\
\hline Total & 10986,33 & 256,09 & 1,000000 & 11812,36 & 263,51 & 1,000000 \\
\hline
\end{tabular}

Bond-Buckup 2004). Embora ocorra um declínio no grau médio de repleção nos outros horários, não foi observado período de descanso alimentar, semelhante ao observado em Callinectes danae (Branco \& Verani 1997). Isso sugere que as espécies se alimentem em todos os horários do dia, ao invés de restringi- rem-se a apenas um período de alimentação.

Os estômagos cheios revelam o horário de alimentação preferencial da espécie, pois indicam que o animal se alimentou recentemente (WILLIAMS 1981), ou ainda que o animal atinge repleção estomacal mais de uma vez por turno (HILL 1976). 


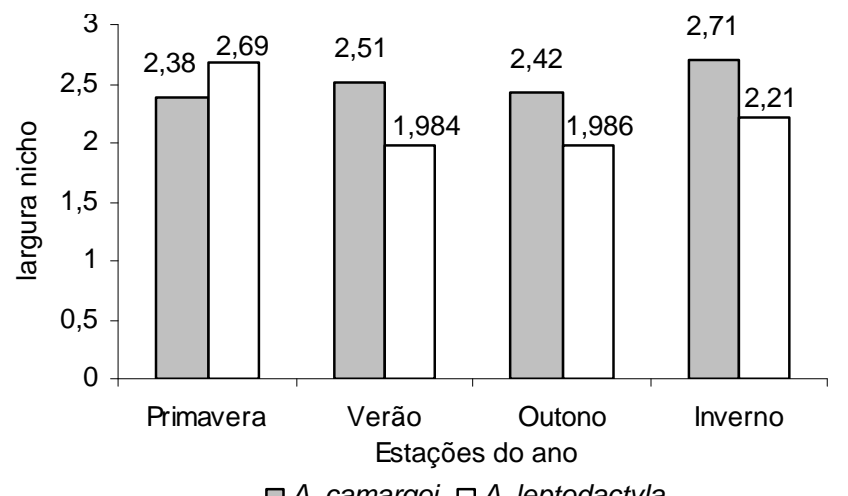

Figura 5. Aegla camargoi e Aegla leptodactyla. Largura do nicho trófico de Levins (números acima da barra), dos indivíduos amostrados no Rio da Divisa, São José dos Ausentes, Rio Grande do Sul, na primavera/2001, verão, outono e inverno/2002.

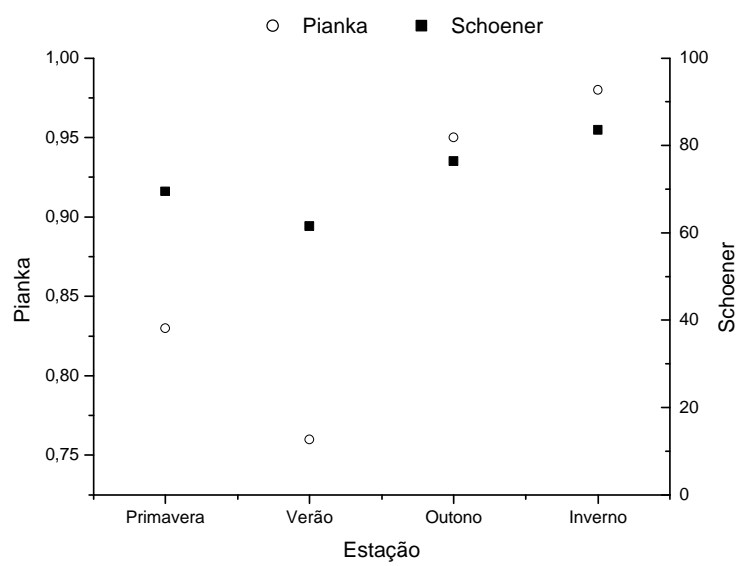

Figura 6. Aegla camargoi e Aegla leptodactyla. Sobreposição do nicho trófico, segundo a medida de Pianka (eixo à esquerda) e de Schoener (eixo à direita), entre os indivíduos amostrados no Rio da Divisa, São José dos Ausentes, Rio Grande do Sul, na primavera/2001, verão, outono e inverno/2002.

Para as espécies estudadas, não se detectou um turno preferencial de forrageamento, pois, a análise estatística não mostrou diferenças significativas entre os horários amostrados e os estômagos estiveram repletos ou parcial mente repletos em todos os horários. Por outro lado, os picos de horários de atividade alimentar observados às $18 \mathrm{~h}$ para Aegla leptodactyla e às $12 \mathrm{~h}$ para A. camargoi, sugerem que estas espécies possam estar alternando seus horários de al imentação.

Os resultados indicaram que as macrófitas e os insetos imaturos são recursos básicos na dieta de $A$. camargoi e $A$. leptodactyla. Ao analisar os val ores de FO das ordens de Insecta, agrupados com os valores de partes de insetos não-identificados, observou-se que esta é a segunda categoria mais impor- tante para as espécies $(58,6 \%$ em A. camargoi, e $80 \%$ em A. leptodactyla), não considerando o item material digerido, que provavelmente seja derivado das partes moles dos insetos, das macrófitas e/ou al gas.

O componente areia, já documentado em estômagos de aeglídeos por BUENO \& BOND-BUCKUP (2004), pode ter sido ingerido ao acaso. Todavia, existe a possibilidade da ingestão ter sido voluntária, se considerarmos que o substrato contém muitos microorganismos associados, que podem servir como fonte alimentar (WEAR \& HADDON 1987, BRANCo \& VERANI 1997, BUENO \& BOND-BUCKUP 2004), tais como os crustáceos Ostracoda, e os Oligochaeta, estes verificados somente nos estômagos de A. camargoi . Como A. leptodactyla também ingeriu areia, não se descarta a hipótese de que os Ostracoda e Oligochaeta pudessem estar digeridos em seus estômagos, sendo então considerados material digerido. Também existe a possibilidade de que a areia estivesse auxiliando a trituração do alimento no estômago, fato esse observado em Callinectes ornatus Ordway, 1863 (Mantelatto \& Christofoletti 2001).

As macrófitas mostraram ser o componente alimentar mais importante tanto na dieta de A. camargoi como na de A. leptodactyla, corroborando as observações de KAPUSTA \& Bemvenuti (1998), que destacaram a alta qualidade energética desses vegetais para Callinectes sapidus Rathbun, 1895. Nos aeglídeos, no entanto, não se conhece, ainda, o papel das macrófitas na dieta das espécies e como se processa a digestão do vegetal. A relação entre as espécies estudadas e as macrófitas corroboram as observações realizadas em A. laevis laevis (BAhamonde \& Lopez 1961) e A. platensis e A. ligulata (Bueno \& Bond-BUCKUP 2004). Como nos estômagos dos indivíduos analisados as macrófitas já apresentavam um certo grau de trituração, e, além disso, não eram ingeridas inteiras, mas sim em partes, não foi possível identificar a família à qual pertencem. Contudo, é possível que as macrófitas pertençam à família Podostemonaceae, já que as duas espécies de aeglídeos são encontradas no Rio da Divisa em lajes submersas, cobertas por essas plantas (Noro \& Buckup 2002).

A categoria alimentar material digerido foi o segundo item mais importante na dieta de A. camargoi e A. leptodactyla, sugerindo que os animais alimentaram-se em um período relativamente curto de tempo. De maneira semelhante, em Aegla platensis, o material digerido também representou o segundo item mais importante, e em Aegla ligulata, o material digerido foi o item mais importante para os adultos (BUENo \& BONDBuckUp 2004). Como essa categoria foi encontrada em todos os horários de amostragem, pode-se inferir que os animais alimentam-se em todos os horários e apresentam uma rápida digestão. O material digerido pode derivar de itens de origem predominantemente animal, como oligochaeta e insetos, já observados como presas de outras espécies de Aeglidae (BAHAmonde \& Lopez 1961, Rodrigues \& Hebling 1978, Bueno \& BondBuckup 2004) o que explicaria a pequena importância (IA) destes itens nos estômagos das espécies. 
As partes de aeglídeos encontradas com certa freqüência nos estômagos, como em A. platensis (BUENo \& Bond-Buckup 2004), podem estar associadas à necessidade de reposição de íons cál cio durante a muda, ou por predação (WILLIAMs 1982) e possivelmente sejam provenientes da ingestão da própria exúvia do animal. Por outro lado, o canibal ismo vem sendo observado entre os Brachyura, como em Carcinus maenas Linnaeus 1758, Portunus pelagicus Linnaeus 1766, e em juvenis de Cancer magister Dana 1852 (Williams 1982, Elner 1981, Stevens et al. 1982, respectivamente).

As categorias alimentares metacercária de Platyhelminthes não-identificado e Temnocephala sp, o último só encontrado nos estômagos de $A$. leptodactyla, mostraram uma pequena importância alimentar (IA) na dieta natural das duas espécies, indicando uma ingestão acidental desses itens, uma vez que Temnocephala é epibionte de várias espécies de Aegla (BAhamonde \& Lopez 1961, Amato et al. 2003).

As Algae e os Pisces podem ter sido sub-estimadas em suas importâncias relativas. No caso das Algae, Noro \& Buckup (2002) verificaram que no Rio da Divisa existem vários grupos, principal mente al gas Rodhophyta aderidas ao substrato rochoso, constituindo fonte alimentar disponível. Em relação aos peixes, alguns autores registraram observações de indivíduos de Aegla ingerindo peixes sal monídeos (BuRns 1972, BAHAMONDE \& Lopez 1961). Bueno \& Bond-Buckup (2004), de modo semeIhante, encontraram escamas de peixes nos estômagos de indivíduos de A. platensis e A. ligulata.

Variações sazonais na dieta das duas espécies podem refletir variações na disponibilidade de presas durante o ano. Branco \& Verani (1997) verificam que, de fato, a dieta de C. danae mostrou variações durante as amostragens, relacionadas com à disponibilidade de presas no ambiente durante as estações. As diferenças na composi ção dos itens alimentares também podem estar relacionadas com as diferentes necessidades nutricionais das espécies ao longo do ano (Rosas et al. 1994), como para reprodução e muda, entre outros.

A maior importância dos insetos, tanto para A. camargoi como para A. leptodactyla, no período do outono e inverno, corresponde à época do ano em que seus estágios imaturos são mais abundantes nos cursos d'água. Entretanto, a importância dos insetos, de modo geral, é baixa (entre 0,04 e 0,20 para A. camargoi, e entre 0,07 e 0,17 para A. leptodactyla), e pode ser explicada pelo fato desses animais serem pouco volumosos, atingindo baixos valores em pontos, e suas partes moles serem facilmente digeridas, podendo ter sido avaliadas como material digerido.

Os val ores das larguras do nicho trófico foram mais al tos para A. camargoi em todas as estações, à exceção da primavera, indicando que essa espécie alimenta-se de um maior espectro alimentar. Por sua vez, A. leptodactyla, mostrou o menor valor de largura de nicho trófico no outono, coincidindo com o seu período de reprodução, que se estende desde o final do outono até o início da primavera (Noro \& Buckup 2002).
Neste estudo, as medidas de sobreposição de PIANKA (1973) e de SCHOENER (1970) mostraram a mesma tendência, apresentando altos val ores de sobreposição entre as duas espécies em todas as estações, com um pico no inverno, quando o compartiIhamento de recursos foi quase total. No verão a sobreposição de nicho apresentou, por outro lado, os menores valores, indicando que as duas espécies alimentaram-se diferentemente: A egla camargoi não ingeriu Insecta Coleoptera e Diptera, eA egla leptodactyla não ingeriu escamas de pisces e Insecta Ephemeroptera. O uso de diferentes índices mostrou resultados similares, assim como verificado no estudo de AlBERTONI et. al. (2003), que utilizaram diversos índices de sobreposição em três espécies simpátricas de Penaeidae e Palaemonidae, e obtiveram resultados semel hantes: uma al ta sobreposi ção na dieta das duas espécies de Penaeidae, e pequena sobreposição quando comparada com a de Palaemonidae.

Apesar da alta sobreposição observada entre as espécies de aeglídeos, não se pode afirmar que esteja havendo competição por recursos. As espécies talvez ocupem nichos semelhantes, porém não idênticos, de modo a explorarem os recursos alimentares em quantidade ou freqüência distintas (ABrams 1980). Ou ainda, al gum dos recursos al imentares pode ser abundante (Colwell \& Futuyma 1971), não havendo necessidade de competir pelo alimento.

A grande diversidade de recursos alimentares disponíveis no ambiente, (GBB, comunicação pessoal) e o amplo espectro al imentar encontrado nos estômagos das duas espécies, sugere que estas compartilham tais recursos, corroborando os resultados obtidos da análise do conteúdo estomacal e da repleção estomacal, que revelou estômagos médio-cheios nas quatro estações do ano.

Os resultados sugerem que tanto $A$. camargoi como $A$. leptodactyla possuem hábito alimentar omnívoro generalista e oportunista, com preferência por alimento de origem vegetal. São consideradas generalistas, pois consomem vegetação macrófita, ampla variedade de imaturos de insetos e outros integrantes da epifauna e infauna. A clara preferência de A. leptodactyla por macrófitas, com el evado valor de IA, evidencia o oportunismo da espécie, identificado pelo seu predomínio nos ambientes com maior abundância de Podostemonaceae. A espécie A. camargoi , por sua vez, também apresenta comportamento al imentar omnívoro, sugerindo que as espécies possam estar explorando diferentes microhabitats.

\section{A GRA DECIMENTOS}

A CAPES pela concessão da bolsa mestrado a TCS. Ao CNPq, pela concessão da bolsa produtividade a GBB. Aos colegas do laboratório de crustáceos da UFRGS, pelo auxílio nas pesquisas, tanto em campo como em laboratório, em especial à Alessandra A. de P. Bueno, pelo auxílio nas análises estatísiticas. Ao CPG / PPG BAN da UFRGS. Ao prefeito de São Jose dos Ausentes, Sr. Carlos Búrigo, pelo auxílio no transporte até o local de coleta. 


\section{REFERÊNCIAS BIBLIOGRÁ FICAS}

Abrams, P. 1980. Some comments on measuring niche overlap. Ecology, Durhan, 61 (1): 44-49.

Albertonı, E.F.; C. Palma-Silva \& F.A. Esteves. 2003. Overlap of dietary niche and electivity of three shrimp species (Crustacea, Decapoda) in a tropical coastal lagoon (Rio de Janeiro, Brazil). Revista Brasileira de Zoologia, Curitiba, 20 (1): 135-140.

Amato, J.F.R.; S.B. Amato \& L.C.C. Daudt. 2003. New species of Temnocephala Blanchard (Platyhelminthes, Temnocephalida) ectosymbiont on Aegla serrana Buckup \& Rossi (Crustacea, Anomura) from southern Brazil. Revista Brasileira de Zoologia, Curitiba, 20 (3): 493-500.

BAHAMONDE, N. \& M.T. Lopez. 1961.Estudios biológicos en la populación de Aegla laevis laevis (Latreille) de el Monte (Crustacea, Decapoda, Anomura). Investigaciones zoológicas chilenas, Santiago, 7: 19-58.

Bond-Buckup, G. 2003. Família Aeglidae, p. 21-116. In: G.A.S. Melo (Ed.). Manual de identificação dos Crustacea Decapoda de água doce do Brasil. São Paulo, Ed. Loyola, 429p.

Bond-Buckup, G. \& L. Buckup. 1994. A Família Aeglidae (Crustacea, Decapoda Anomura). Arquivos de Zoologia do Estado de São Paulo, São Paulo, 32 (4): 159-346.

Branco, J.O. \& J.R. Veranı. 1997. Dinâmica da al imentação natural de Callinectes danae Smith (Decapoda, Portunidae) na Lagoa da Conceição, Florianópolis, Santa Catarina, Brasil. Revista Brasileira de Zoologia, Curitiba, 14 (4): 1003-1018.

Brogim, R.A. \& P.C. LanA. 1997. Espectro alimentar de Aratus pisonii, Chasmagnathus granulata eSesarma rectum (Decapoda: Grapsidae) em um manguezal na Baía de Paranaguá, Paraná. Iheringia, Porto Alegre, 83: 35-43.

Bueno, A.A.P. \& G. Bond-Buckup. 2004. Natural diet of Aegla platensis Schmitt and Aegla ligulata Bond-Buckup \& Buckup (Crustacea, Decapoda, Aeglidae) from Brazil. Acta Limnologica Brasiliensis, São Paulo, 16 (2): 115-127.

Burns, J.W. 1972. The distribution and life history of the south american freshwater crabs (Aegla) and their role in trout streams and lakes. Transactions of the American Fisheries Society, Bethesda, 101 (4): 595-607.

Charvet, S.; A. Kosmala \& B. Statzner. 1998. Biomonitoring through biological traits of benthic macroinvertebrates: perspectives for a general tool in stream management. Archives Hydrobiology, Amsterdan, 142 (4): 415-432.

ColwelL, R.K. \& D.J. FutuymA. 1971. On themeasurement of niche breath and overlap. Ecology, Durhan, 52 (4): 567-576.

ELnER, R.W. 1981. Diet of green crab Carcinus maenas (L.) from Port Hebert, Southwestern Nova Scotia. Journal of Shellfish Research, New York, 1: 89-94.

Haefner JR., P.A. 1990. Natural diet of Callinectes ornatus (Brachyura: Portunidae) in Bermuda. Journal of Crustacean Biology, Kansas, 10 (2): 236-246.
HILL, B.J. 1976. Alimentação natural, taxa de desobstrução do estômago e atividade do caranguejo Scylla serrata. Marine Biology, Berlim, 34: 109-116.

Hollows, J.W.; C.R. Townsend \& K.J. Collier. 2002. Diet of Paranephrops zealandicus in bush and pasture streams: insights from stable isotopes and stomach analysis. New Zealand Journal of Marine Freshwater Research, Wellington, 36: 129-142.

HURLBERT, S.H. 1978.The measurement of niche overlap and some relatives. Ecology, Durhan, 59 (1): 67-77.

Kapusta, S.C. \& C.E. Bemvenutı. 1998. Atividade nictemeral de alimentação de juvenis de Callinectes sapidus Rathbun, 1895 (Decapoda: Portunidae) numa pradaria de Ruppia maritima L. e num plano não vegetado, numa enseada estuarina da Lagoa dos Patos, RS, Brasil. Nauplius, Rio Grande, 6: 41-52

KAWAKAMI, E. \& G. VAZzoler. 1980. Método gráfico e estimativa de índice alimentar aplicado no estudo de alimentação de peixes. Boletim do Instituto Oceanográfico, São Paulo, 29 (2): 205-207.

KREBS, C.J. 1989. Ecological methodology. New York, Harper \& Row, 654p.

LeVINS, R. 1968. Evolution in changing environments. New Jersey, Princeton University Press, IX $+120 p$.

Mantelatto, F.L.M. \& M. Petracco. 1997. Natural diet of the crab Hepatus pudibundus (Brachyura: Callapidae) in Fortaleza Bay, Ubatuba (SP), Brazil. J ournal of Crustacean Biology, Kansas, 17 (3): 440-446.

Mantelatto, F.L.M. \& R.A. Christofoletti. 2001. Natural feeding activity of the crab Callinectes ornatus (Portunidae) in Ubatuba Bay (São Paulo, Brasil): influence of season, Sex, size and molt stage. Marine Biology, Berlim, 138: 585-594.

Nimer, E. 1989. Climatologia do Brasil. Rio de Janeiro, IBGE, $421 \mathrm{p}$.

Noro, C.K. \& L. Buckup. 2002. Biologia reprodutiva e ecologia de Aegla leptodactyla Buckup \& Rossi (Crustacea, Anomura, Aeglidae). Revista Brasileira de Zoologia, Curitiba, 19 (4): 1063-1075.

Pianka, E.R. 1973 The structure of lizard communities. Annual Review of Ecology and Systematics, Stanford, 4: 53-74.

Rodrigues, W. \& N.J. Hebling. 1978. Estudos biológicos em Aegla perobae Hebling \& Rodrigues, 1977 (Decapoda, Anomura). Revista. Brasileira de Biologia, Rio de Janeiro, 38 (2): 383390.

Rosas, C.; E. Lazaro-Chavez \& F. Bückle-Ramirez. 1994. Feeding habits and food niche segregation of Callinectes sapidus, $C$. rathbunae $\mathrm{C}$. similis in a subtropical coastal lagoon of the Gulf of Mexico. Journal of Crustacean Biology, Kansas, 14 (2): 371-382.

SCHOENER, T. 1970. Nonsynchronous spatial overlap of lizards in patchy habitats. Ecology, Durhan, 51 (3): 408-418.

Stevens, B.G.; D.A. Armstrong \& R. Cusimano. 1982. Feeding habits of the dungeness crab Cancer magister as determined by the Index of Relative Importance. Marine Biology, 
Berlim, 72: 135-145.

WeAR, R.G. \& M. HADDon. 1987. Natural diet of the crab Ovalipes catharus (Crustacea, Portunidae) around Central and Northen New Zealand. Marine Ecology Progress Series, Amelinghausen, 35: 39-49.

WilLiAMS, M.J. 1981. Methods for analysis of natural diet in portunid crabs (Crustacea: Decapoda: Portunidae). Journal of Experimental Marine Biology and Ecology, Amsterdan, 52: 103-113.

. 1982. Natural food and feeding in the commercial sand crab Portunus pelagicus Linnaeus, 1766 (Crustacea: Decapoda: Portunidae) in Moreton Bay, Queensland. Journal of Experimental Marine Biology and Ecology, Amsterdan, 59: 165-176.

Recebido em 09.III.2004; aceito em 18.X.2004. 